\title{
Diagnostic Power of Cytokine M-CSF, Metalloproteinase 2 (MMP-2) and Tissue Inhibitor-2 (TIMP-2) in Cervical Cancer Patients Based on ROC Analysis
}

\author{
Emilia Lubowicka ${ }^{1}$ (D) Monika Zbucka-Kretowska ${ }^{2}$ - Iwona Sidorkiewicz ${ }^{2} \cdot$ Monika Zajkowska $^{3}$ - Ewa Gacuta ${ }^{4}$. \\ Andrzej Puchnarewicz ${ }^{5}$ - Lech Chrostek ${ }^{3}$ - Maciej Szmitkowski ${ }^{3}$ - Sławomir Ławicki ${ }^{6}$
}

Received: 6 March 2018 / Accepted: 20 February 2019 /Published online: 28 February 2019

(C) The Author(s) 2019

\begin{abstract}
Macrophage colony-stimulating factor (M-CSF), matrix metalloproteinase-2 (MMP-2) and its specific tissue inhibitor (TIMP-2) may play an important role in the pathogenesis of cancer disease. We investigated the plasma levels and diagnostic power (ROC curve analysis) of M-CSF, MMP-2, TIMP-2 and tumor markers CA 125 and SCC-Ag in cervical cancer (CC) patients as compared to control group. The study included 89 patients with cervical cancer. The control group consisted of 50 healthy, untreated women. The plasma levels of M-CSF, MMP-2 and TIMP-2 were determined using ELISA, CA 125 and SCC-Ag - by CMIA method. The median levels of M-CSF, TIMP-2, SCC-Ag and CA 125 in the entire group of CC were significantly different than compared to the healthy women group. MMP-2 showed the highest value of sensitivity from all examined parameters (in stage I of CC $-93.10 \%$, II $-82.76 \%$, III and IV $-96.88 \%$, total group $-92.05 \%$ ). The highest specificity was obtained by M-CSF ( $86 \%)$. The area under the ROC curve (AUC) of M-CSF $(0.8051)$ was the largest of all the tested parameters (even higher than commonly used tumor markers) in the group of cervical cancer. The combination of M-CSF, MMP-2 or TIMP2 with SCC antigen resulted in an increase AUCs in all cases $(0.8760 ; 0.7880 ; 0.8081$; respectively). The findings of this study suggest the usefulness of all examined parameters in the diagnostics of CC patients. Out of the tested substances, M-CSF also appears to be the best candidate for cancer diagnostics in all stages of the disease, based on ROC analysis.
\end{abstract}

Keywords M-CSF $\cdot$ MMP-2 $\cdot$ TIMP-2 $\cdot$ Cervical cancer $\cdot$ Tumor markers

Emilia Lubowicka

emila_lubowicka@wp.pl

Monika Zbucka-Kretowska

monikazbucka@wp.pl

Iwona Sidorkiewicz

iwona.sidorkiewicz@gmail.com

Monika Zajkowska

monika@zajkowska.com

Ewa Gacuta

sunnyeve@wp.pl

Andrzej Puchnarewicz

ampuchn@wp.pl

Lech Chrostek

chrostek@umb.edu.pl

Maciej Szmitkowski

msz@umb.edu.pl
Sławomir Ławicki

slawicki@umb.edu.pl

1 Department of Esthetic Medicine, Medical University of Bialystok, Akademicka 3, 15-267 Bialystok, Poland

2 Department of Reproduction and Gynecological Endocrinology, Medical University of Bialystok, 15-276 Bialystok, Poland

3 Department of Biochemical Diagnostics, Medical University of Bialystok, 15-269 Bialystok, Poland

4 Department of Perinatology, Medical University of Bialystok, 15-276 Bialystok, Poland

5 Department of Urology, Provincial Hospital, 15-278 Bialystok, Poland

6 Department of Population Medicine and Civilization Diseases Prevention, Medical University of Bialystok, 15-269 Bialystok, Poland 


\section{Introduction}

Cancer of the uterine cervix is the leading cause of death among gynecological cancers in developing countries and the fourth principal cause of cancer-related death in women worldwide [1]. High-risk human papillomavirus (HPV) infection is considered the most important risk factor associated with the development of this tumor, and it is present in $99.7 \%$ of invasive cervical tumors worldwide including essentially all squamous cell cancers and adenocarcinomas [2].

Reliable methods for the accurate identification of both the presence and severity of cervical intraepithelial neoplasia (CIN) and extent of spread of invasive carcinomas of the cervix (IC) are critical. The primary objective of cervical cancer screening is to detect cervical intraepithelial neoplasia (CIN 3 ) sufficiently early so that it can be treated to prevent the development of cancer. Screening based on cytological testing (commonly known as the smear test or Pap test) is still the most important diagnostic technique for detecting preinvasive cervical cancer [3]. Despite the prevention and early detection of cervical cancer, it is still the second leading cause of cancer- related death in young women worldwide. Further research is thus required to select prognostic biomarkers and therapeutic targets. No marker is completely specific, and therefore, diagnostic testing must be used in conjunction with morphological and clinical findings.

Macrophage colony-stimulating factor (M-CSF) is a hematopoietic growth factor that stimulates the proliferation and differentiation of monocytes to macrophages. In inflammation M-CSF induces macrophages to secrete cytokines and proteases, thereby enhancing the macrophages' ability to combat microbial infections [4]. Increased expression of M-CSF and its receptor is correlated with poor prognosis in breast, ovarian and prostate cancer [5-11]. M-CSF has mainly been studied in breast carcinomas, where it is commonly expressed $[12,13]$. Several other candidate markers have also been implicated, paricularly those involved in tumor invasion. A few studies have connected matrix metalloproteinases (MMPs), particularly gelatinases (MMP-2 and MMP-9), to tumor angiogenesis and growth [14]. MMP-2 and MMP-9 have been shown to be upregulated in angiogenic lesions [15]. The activity of the metalloproteinases is controlled by macroglobulins and, predominantly, tissue inhibitors of metalloproteinases (TIMPs). TIMP-2 functions include binding and inhibiting the proteolytic activity of MMPs and the activation of proMMP-2 [16]. Therefore, changes in TIMP-2 and MMP-2 levels would also determine whether TIMP-2 role is promoting or inhibitory. The main activation route of MMP-2 on the cell surface is by the formation of a molecular complex containing MMP-2, membrane type 1-matrix metalloproteinase 1 (MT1-MMP) and TIMP-2 [17]. Imbalance between the activity of MMPs and TIMPs has been attributed to the ability of cancer cells to migrate [18]. TIMP-2 correlates with poor prognosis in many types of cancer [19-21].

The aim of this study was to determine plasma levels of MCSF, MMP-2 and TIMP-2 in comparison to CA 125 and SCC-Ag concentrations in patients with cervical cancer in relation to the control group (healthy subjects). Additionally, the diagnostic criteria: sensitivity (SE) and specificity (SP) were defined in the study. Furthermore, the study defined the receiver-operating characteristics (ROC) curve for all the tested parameters alone and in combination with tumor markers (CA 125 and SCC-Ag). The data obtained in the present study may be used in evaluating the usefulness (especially diagnostic power) of the tumor marker panel in the diagnostics of cervical cancer patients.
Table 1 Characteristics of cervical cancer patients and control group (healthy women)

\begin{tabular}{llll}
\hline Study group & & & Number of patients \\
\hline Tested Group & Cervical cancer patients & Squamous cell carcinoma & 89 \\
& Median age (range) & & $47(25-61)$ \\
& Tumor stage & I & 29 \\
& & II & 28 \\
& III and IV & 32 \\
Control group & & \\
& - premenopausal & & 69 \\
& - - -ealthy womenopausal & & 20 \\
& Median age (range) & & 50 \\
& Menopausal status: & & $42(22-61)$ \\
& - premenopausal & & 39 \\
& - postmenopausal & 11
\end{tabular}


Table 2 Plasma levels of tested parameters and CA 125 and SCC-Ag in patients with cervical cancer and in control group

\begin{tabular}{|c|c|c|c|c|c|}
\hline Groups tested & M-CSF (pg/mL) & MMP-2 (ng/mL) & TIMP-2 (ng/mL) & SCC-Ag (U/mL) & CA $125(\mathrm{U} / \mathrm{mL})$ \\
\hline \multicolumn{6}{|c|}{ Cervical cancer (median, range) } \\
\hline Stage I & $422.55^{\mathrm{a}}(102.15-2513.75)$ & $200.00(124.84-352.00)$ & $70.00^{\mathrm{a}}(40.00-160.00)$ & $1.29(0.38-2.20)$ & $14.40(6.60-49.60)$ \\
\hline Stage II & $510.55^{\mathrm{a}}(95.26-1304.80)$ & $218.00(129.80-379.00)$ & $71.50(26.93-120.00)$ & $1.20(0.45-5.90)$ & $17.40^{\mathrm{a}}(4.40-77.41)$ \\
\hline Stages III and IV & $578.50^{\mathrm{a}}(113.05-2511.95)$ & $221.00^{\mathrm{a}}(140.50-351.96)$ & $84.60^{\mathrm{b} / \mathrm{c}}(50.00-156.00)$ & $1.20(0.30-14.10)$ & $25.65^{\mathrm{a} / \mathrm{b}}(6.34-120.10)$ \\
\hline Total group & $510.55^{\mathrm{a}}(95.23-2513.75)$ & $214.00(124.84-379.00)$ & $76.00^{\mathrm{a}}(26.93-160.00)$ & $1.20^{\mathrm{a}}(0.30-14.10)$ & $17.99^{\mathrm{a}}(4.40-120.10)$ \\
\hline \multicolumn{6}{|c|}{ Control groups (median, range) } \\
\hline Healthy women & $251.50(119.63-935.29)$ & $202.95(24.30-397.20)$ & $87.25(42.50-132.50)$ & $0.75(0.40-1.60)$ & $11.70(3.50-36.60)$ \\
\hline
\end{tabular}

a Statistically significant when patients with CC compared with healthy women.

b Statistically significant when patients with CC stages III and IV compared with patients with CC stage I

${ }^{c}$ Statistically significant when patients with CC stages III and IV compared with patients with CC stage II

\section{Material and Methods}

\section{Human Subjects}

Table 1 shows the tested and control group. The study comprised 89 patients with invasive primary carcinoma of the uterine cervix who were referred to the Department of Gynecology, Bialystok Medical University Teaching
Hospital, Poland, between 2012 and 2016. Clinical stages and histological classification based on the criteria of the International Federation of Gynecology and Obstetrics (FIGO) were established in all cases. Written consent including participants' own statements regarding their medical history (i.e. data related to reproductive history, personal or family history of cancer, general health issues - hospitalization or surgery, use of medication) and lifestyle habits including
Table 3 Diagnostic criteria of tested parameters and in combined analysis with CA 125 and SCC-Ag in cervical cancer patients

\begin{tabular}{|c|c|c|c|c|c|}
\hline \multirow[t]{2}{*}{ Tested parameters } & \multirow{2}{*}{$\begin{array}{l}\text { Diagnostic } \\
\text { criteria }(\%)\end{array}$} & \multicolumn{4}{|c|}{ Cervical cancer } \\
\hline & & Stage I & Stage II & Stages III and IV & Total \\
\hline \multirow[t]{2}{*}{$\mathrm{M}-\mathrm{CSF}$} & SE & 51.72 & 75.00 & 78.13 & 69.41 \\
\hline & SP & 86.00 & 86.00 & 86.00 & 86.00 \\
\hline \multirow[t]{2}{*}{ MMP-2 } & SE & 93.10 & 82.76 & 96.88 & 92.05 \\
\hline & SP & 58.00 & 58.00 & 58.00 & 58.00 \\
\hline \multirow[t]{2}{*}{ TIMP-2 } & SE & 17.24 & 17.86 & 59.38 & 32.18 \\
\hline & SP & 60.00 & 60.00 & 60.00 & 60.00 \\
\hline \multirow[t]{2}{*}{ CA 125} & SE & 62.07 & 82.14 & 96.88 & 80.00 \\
\hline & SP & 68.00 & 68.00 & 68.00 & 68.00 \\
\hline \multirow[t]{2}{*}{ SCC-Ag } & SE & 75.86 & 78.57 & 78.13 & 81.18 \\
\hline & $\mathrm{SP}$ & 74.00 & 74.00 & 74.00 & 74.00 \\
\hline \multirow[t]{2}{*}{$\mathrm{M}-\mathrm{CSF}+\mathrm{CA} 125$} & SE & 79.31 & 93.10 & 100.00 & 91.76 \\
\hline & $\mathrm{SP}$ & 66.00 & 66.00 & 66.00 & 66.00 \\
\hline \multirow[t]{2}{*}{ MMP-2 + CA 125} & SE & 96.55 & 100.00 & 100.00 & 98.88 \\
\hline & SP & 30.00 & 30.00 & 30.00 & 30.00 \\
\hline \multirow[t]{2}{*}{ TIMP-2 + CA 125} & SE & 68.97 & 85.71 & 100.00 & 85.39 \\
\hline & SP & 28.00 & 28.00 & 28.00 & 28.00 \\
\hline \multirow[t]{2}{*}{$\mathrm{M}-\mathrm{CSF}+\mathrm{SCC}-\mathrm{Ag}$} & SE & 86.21 & 92.86 & 93.75 & 91.76 \\
\hline & SP & 66.00 & 66.00 & 66.00 & 66.00 \\
\hline \multirow[t]{2}{*}{ MMP-2 + SCC-Ag } & SE & 96.55 & 96.43 & 100.00 & 96.63 \\
\hline & SP & 36.00 & 36.00 & 36.00 & 36.00 \\
\hline \multirow[t]{2}{*}{ TIMP-2 + SCC-Ag } & SE & 75.86 & 79.31 & 87.50 & 82.02 \\
\hline & SP & 30.00 & 30.00 & 30.00 & 30.00 \\
\hline
\end{tabular}

Abbreviations: $C A$, cancer antigen; $M M P-2$, matrix metalloproteinase-2; TIMP-2, tissue inhibitor of metalloproteinase-2; $M-C S F$, macrophage-colony stimulating factor; $S E$, sensitivity; $S P$, Specificity 
smoking was obtained from all the subjects. None of the patients had received chemo- or radiotherapy before blood sample collection. Pretreatment staging procedures included physical and blood examinations, ultrasound scanning and chest X-rays. In addition, CT (computed tomography) scans or MRI (magnetic resonance imaging) were performed where necessary. The control group included 50 healthy and untreated women (aged 22-61 years). In these women cervical smears were examined by a gynecologist prior to blood collection. In addition, a reproductive organ ultrasound scan was performed where necessary. All subjects had undergone annual checkups (laboratory tests, USG, chest x-ray, cervical cytology screening, mammography). The study was approved by the local Ethics Committee (R-I-002/239/2014) and all the patients gave their informed consent for study participation.

\section{Plasma Collection and Storage}

Venous blood samples were collected from each patient. Blood was collected into heparin sodium tubes, centrifuged $3500 \mathrm{rpm}$ for $20 \mathrm{~min}$ to obtain plasma samples, and stored at $-85^{\circ} \mathrm{C}$ until assayed.

\section{Measurement of M-CSF, MMP-2, TIMP-2, CA 125 and SCC-Ag}

The examined parameters (MMP-2, TIMP-2, and M-CSF) were measured with enzyme-linked immunosorbent assay (ELISA) (Quantikine Human M-CSF Immunoassay; R\&D

Table 4 Diagnostic criteria of ROC curve for tested parameters and CA 125 and SCC-Ag in total group of CC

\begin{tabular}{lclll} 
Tested parameters & AUC & SE & $95 \%$ C.I. (AUC) & $p(\mathrm{AUC}=0.5)$ \\
\hline ROC criteria in cervical cancer (total) & & \\
M-CSF & 0.8051 & 0.0383 & $0.730-0.880$ & $<0.001$ \\
MMP-2 & 0.5882 & 0.0566 & $0.477-0.699$ & 0.1195 \\
TIMP-2 & 0.6186 & 0.0532 & $0.514-0.723$ & 0.0257 \\
CA 125 & 0.7340 & 0.0461 & $0.644-0.824$ & $<0.001$ \\
SCC-Ag & 0.7866 & 0.0383 & $0.711-0.862$ & $<0.001$ \\
M-CSF + CA 125 & 0.8006 & 0.0376 & $0.727-0.874$ & $<0.001$ \\
MMP-2 + CA 125 & 0.5963 & 0.0563 & $0.486-0.707$ & 0.0874 \\
TIMP-2 + CA 125 & 0.6166 & 0.0537 & $0.511-0.722$ & 0.0299 \\
M-SCF + SCC-Ag & 0.8760 & 0.0296 & $0.818-0.934$ & $<0.001$ \\
MMP-2 + SCC-Ag & 0.7880 & 0.0381 & $0.713-0.863$ & $<0.001$ \\
TIMP-2 + SCC-Ag & 0.8081 & 0.0370 & $0.736-0.881$ & $<0.001$ \\
\hline
\end{tabular}

$p$ - statistically significantly larger AUCs compared to $\mathrm{AUC}=0.5$

Abbreviations: $R O C$, receiver-operating characteristics; $C A$, cancer antigen; $A U C$, area under the ROC curve; $S E$, standard error; $C I$, confidence interval; $M-C S F$, macrophage-colony stimulating

factor; $M M P-2$, matrix metalloproteinase-2; TIMP-2, tissue inhibitor of metalloproteinase-2 systems, Abingdon, United Kingdom), according to the manufacturer's instructions. This assay employs the quantitative sandwich enzyme immunoassay technique. Plasma concentrations of SCC-Ag and CA 125 were measured by chemiluminescent microparticle immunoassay (CMIA)

Table 5 Diagnostic criteria of ROC curve for tested parameters and CA 125 and SCC-Ag in all stages of CC

\begin{tabular}{|c|c|c|c|c|}
\hline Tested parameters & AUC & SE & 95\% C.I. (AUC) & $p(\mathrm{AUC}=0.5)$ \\
\hline \multicolumn{5}{|c|}{ ROC criteria in cervical cancer (I stage) } \\
\hline $\mathrm{M}-\mathrm{CSF}$ & 0.7109 & 0.0631 & $0.582-0.829$ & 0.0011 \\
\hline MMP-2 & 0.5484 & 0.0658 & $0.421-0.678$ & 0.4223 \\
\hline TIMP-2 & 0.6747 & 0.0622 & $0.562-0.799$ & 0.0064 \\
\hline CA 125 & 0.6429 & 0.0614 & $0.522-0.760$ & 0.0188 \\
\hline SCC-Ag & 0.8022 & 0.0521 & $0.709-0.908$ & $<0.001$ \\
\hline $\mathrm{M}-\mathrm{CSF}+\mathrm{CA} 125$ & 0.8001 & 0.0641 & $0.591-0.839$ & $<0.001$ \\
\hline MMP-2 + CA 125 & 0.5499 & 0.0649 & $0.423-0.677$ & 0.4646 \\
\hline TIMP-2 + CA 125 & 0.6661 & 0.0627 & $0.551-0.796$ & 0.0088 \\
\hline $\mathrm{M}-\mathrm{SCF}+\mathrm{SCC}-\mathrm{Ag}$ & 0.8498 & 0.0440 & $0.768-0.935$ & $<0.001$ \\
\hline MMP-2 + SCC-Ag & 0.7824 & 0.0534 & $0.684-0.891$ & $<0.001$ \\
\hline TIMP-2 + SCC-Ag & 0.8320 & 0.0461 & $0.758-0.932$ & $<0.001$ \\
\hline \multicolumn{5}{|c|}{ ROC criteria in cervical cancer (II stage) } \\
\hline $\mathrm{M}-\mathrm{CSF}$ & 0.8042 & 0.0532 & $0.698-0.912$ & $<0.001$ \\
\hline MMP-2 & 0.5564 & 0.0639 & $0.433-0.679$ & 0.3915 \\
\hline TIMP-2 & 0.6898 & 0.0611 & $0.558-0.799$ & 0.0062 \\
\hline CA 125 & 0.7297 & 0.0571 & $0.604-0.836$ & $<0.001$ \\
\hline SCC-Ag & 0.7973 & 0.0523 & $0.691-0.908$ & $<0.001$ \\
\hline $\mathrm{M}-\mathrm{CSF}+\mathrm{CA} 125$ & 0.8002 & 0.0559 & $0.678-0.910$ & $<0.001$ \\
\hline MMP-2 + CA 125 & 0.5647 & 0.0634 & $0.441-0.688$ & 0.3082 \\
\hline TIMP-2 + CA 125 & 0.6662 & 0.0612 & $0.551-0.782$ & 0.0054 \\
\hline $\mathrm{M}-\mathrm{SCF}+\mathrm{SCC}-\mathrm{Ag}$ & 0.8857 & 0.0410 & $0.809-0.968$ & $<0.001$ \\
\hline MMP-2 + SCC-Ag & 0.7931 & 0.0529 & $0.698-0.901$ & $<0.001$ \\
\hline TIMP-2 + SCC-Ag & 0.8891 & 0.0458 & $0.748-0.930$ & $<0.001$ \\
\hline \multicolumn{5}{|c|}{ ROC criteria in cervical cancer (III and IV stages) } \\
\hline M-CSF & 0.8797 & 0.0428 & $0.796-0.951$ & $<0.001$ \\
\hline MMP-2 & 0.6588 & 0.0601 & $0.538-0.776$ & 0.0098 \\
\hline TIMP-2 & 0.5107 & 0.0668 & $0.388-0.639$ & 0.8124 \\
\hline CA 125 & 0.8114 & 0.0498 & $0.717-0.901$ & $<0.001$ \\
\hline SCC-Ag & 0.7616 & 0.0564 & $0.652-0.879$ & $<0.001$ \\
\hline $\mathrm{M}-\mathrm{CSF}+\mathrm{CA} 125$ & 0.8702 & 0.0443 & $0.781-0.952$ & $<0.001$ \\
\hline MMP-2 + CA 125 & 0.6698 & 0.0592 & $0.550-0.781$ & 0.0039 \\
\hline TIMP-2 + CA 125 & 0.5201 & 0.0649 & $0.398-0.649$ & 0.7505 \\
\hline $\mathrm{M}-\mathrm{SCF}+\mathrm{SCC}-\mathrm{Ag}$ & 0.8885 & 0.0386 & $0.818-0.962$ & $<0.001$ \\
\hline MMP-2 + SCC-Ag & 0.7881 & 0.0515 & $0.687-0.891$ & $<0.001$ \\
\hline TIMP-2 + SCC-Ag & 0.7607 & 0.0543 & $0.660-0.869$ & $<0.001$ \\
\hline
\end{tabular}

$p$ - statistically significantly larger AUCs compared to AUC $=0.5$

Abbreviations: $R O C$, receiver-operating characteristics; $C A$, cancer antigen; $A U C$, area under the ROC curve; $S E$, standard error; $C I$, confidence interval; $M-C S F$, macrophage-colony stimulating

factor; $M M P-2$, matrix metalloproteinase-2; TIMP-2, tissue inhibitor of metalloproteinase-2 
(Abbott, Chicago, IL, USA). The intra-assay coefficient of variation $(\mathrm{CV} \%)$ of $\mathrm{CA} 125$ is reported to be $2.4 \%$ at a mean concentration of $43.50 \mathrm{U} / \mathrm{mL}, \mathrm{SD}=1.1$. SCC-Ag is reported to be $4.3 \%$ at a mean concentration of $1.97 \mathrm{ng} /$ $\mathrm{mL}, \mathrm{SD}=0.085$. M-CSF is reported to be $3.4 \%$ at a mean concentration of $227 \mathrm{pg} / \mathrm{mL}, \mathrm{SD}=7.7$. MMP-2 is reported to be $3.8 \%$ at a mean concentration of $11.20 \mathrm{ng} / \mathrm{mL}, \mathrm{SD}=$ 0.42 , TIMP-2 to be $6.0 \%$ at a mean concentration of $2.90 \mathrm{ng} / \mathrm{mL}, \mathrm{SD}=0.173$. The inter-assay coefficient of variation $(\mathrm{CV} \%)$ of $\mathrm{CA} 125$ is reported to be $3.9 \%$ at a mean concentration of $43.50 \mathrm{U} / \mathrm{ml}, \mathrm{SD}=1.7$. SCC-Ag is reported to be $5.1 \%$ at a mean concentration of $1.97 \mathrm{ng} /$ $\mathrm{mL}, \mathrm{SD}=0.1$. M-CSF to be $3.1 \%$ at a mean concentration of $232 \mathrm{pg} / \mathrm{ml}, \mathrm{SD}=7.3$. MMP-2 is reported to be $6.6 \%$ at a mean concentration of $11.10 \mathrm{ng} / \mathrm{mL}, \mathrm{SD}=0.738$, TIMP2 to be $6.7 \%$ at a mean concentration of $2.79 \mathrm{ng} / \mathrm{mL}$, $\mathrm{SD}=0.188$. The value of intra- and inter-assay CVs were calculated by the manufacturers and enclosed in the reagent kits. The assay does not exhibit cross-reactivity or interference with numerous human cytokines and other growth factors. Duplicate samples were assessed for each patient.

\section{Statistical Analysis}

Statistical analysis was performed using STATISTICA 12.0 PL (StatSoft, Tulsa, OK, USA). Preliminary statistical analysis (Chi-square test) revealed that the tested parameters and tumor marker levels did not follow a normal distribution. Consequently, the Mann-Whitney U test was used for statistical analysis between cancer and control group. Additionally, statistical analysis between groups with different stages of CC was performed using the Kruskal-Wallis test, and a multivariate analysis of various data - with the post-hoc Dwass SteeleCrichlow-Flinger test. Statistically significant differences were defined as comparisons resulting in $p<0.05$. Diagnostic sensitivity (SE) and specificity (SP) were calculated. The cut off values were calculated by Youden's index (as a criterion for selecting the optimum cut-off point) and for each of the tested parameters was as follows: M-CSF - $394.42 \mathrm{pg} /$ $\mathrm{mL}$; MMP-2 - 155.92 ng/mL; TIMP-2 - $82.12 \mathrm{ng} / \mathrm{mL}$; CA 125-13.44 U/mL; SCC-Ag - $0.89 \mathrm{ng} / \mathrm{mL}$. In the analyses of both diagnostic performance (SE, SP) and ROC curve, only healthy subjects were used as a control group. The construction of the ROC curves was performed using the GraphRoc

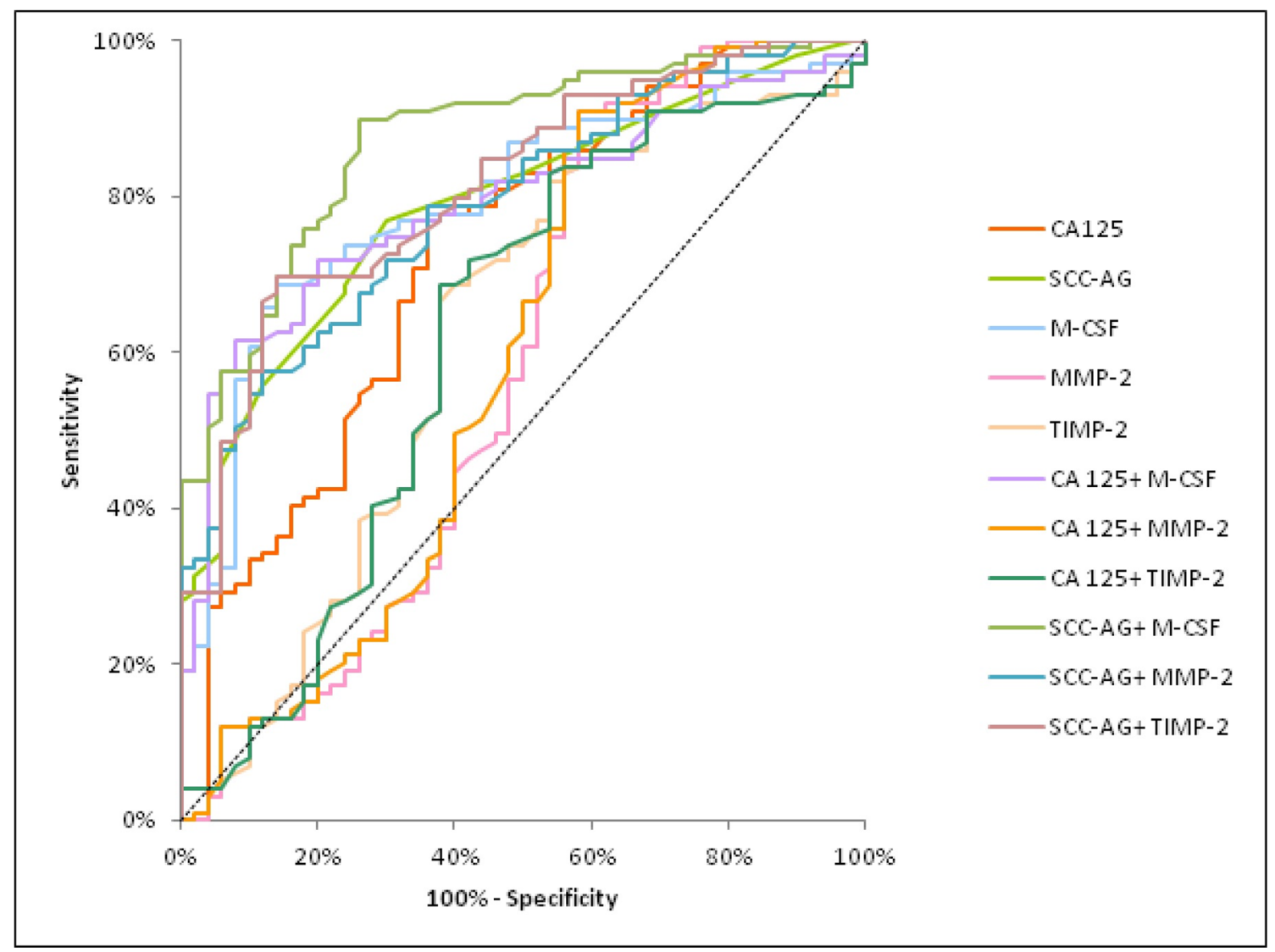

Fig. 1 Diagnostic criteria of ROC curve for examined parameters in combination with CA 125 and SCC-Ag in the cervical cancer group. Abbreviations: ROC, receiver-operating characteristics; M-CSF, macrophage-colony stimulating factor; MMP-2, matrix metalloproteinase-2; TIMP-2, tissue inhibitor of metalloproteinase-2 
program for Windows (Windows, Royal, AR, USA) and the areas under the ROC curve (AUC) were calculated.

\section{Results}

Table 2 presents the median and the range of plasma levels of the investigated parameters and CA 125 and SCC-Ag in the tested groups. The median values for M-CSF $(510.55 \mathrm{pg} / \mathrm{mL})$, similar to those of the commonly accepted tumor markers $\mathrm{CA}$ $125(17.99 \mathrm{U} / \mathrm{mL})$ and SCC-Ag $(1.20 \mathrm{U} / \mathrm{mL})$, in the entire group of $\mathrm{CC}$ were significantly higher compared with the values in healthy subjects $(251.50 \mathrm{pg} / \mathrm{mL} ; 11.70 \mathrm{U} /$ $\mathrm{mL}, 0.75 \mathrm{U} / \mathrm{mL}$, respectively) $(p<0.05)$. Additionally, the median value of TIMP-2 $(76.00 \mathrm{ng} / \mathrm{mL})$ in the total cervical cancer group was significantly lower compared with the values in healthy subjects $(87.25 \mathrm{ng} / \mathrm{mL})(p<0.05)$.

Similarly, we observed statistically significantly higher concentrations of M-CSF in all the analyzed groups in relation to CC stage compared with healthy women $(p<0.05$ in all cases). Additionally, we observed significantly higher concentrations of CA 125 in all the analyzed groups in all stages of cervical cancer (with the exception of stage I) in comparison with healthy women. Significantly higher concentrations of MMP-2 in stages III and IV were also observed $(p<0.05)$. Furthermore, significantly lower concentrations of TIMP-2 in stage I were also noticed $(p<0.05)$.

We also observed significantly higher concentrations of TIMP-2 in stages III and IV of cancer compared with stage I or II (TIMP-2: I vs. III and IV $p<0.05$ and II vs. III and IV $p<0.05$ ). Furthermore, we detected significantly higher plasma levels of CA 125 when stages III and IV was compared to stage I $(p<0.05)$.

Diagnostic criteria for tumor markers are sensitivity (SE) and specificity (SP) (Table 3). We indicated that the SE of the tested cytokines in the total cancer group was the highest for MMP-2 (92.05\%). The combined use of M-CSF, MMP-2 or TIMP-2 with the commonly accepted tumor markers (antigen SCC and CA 125) resulted in an increase in the sensitivity range in the total CC group. Maximum diagnostic sensitivity (98.88\%) was obtained for the combination of MMP-2 with CA 125. Among all parameters, the highest $\mathrm{SE}$ in all stages of cancer was observed for MMP-2 (in stage I of CC - 93.10\%, in stage II of CC $82.76 \%$, and in stages III and IV of CC $-96.88 \%$ ). The combined use of the tested parameters and CA 125 and SCC-Ag resulted in an increase of SE in every stage of CC. A maximum range was

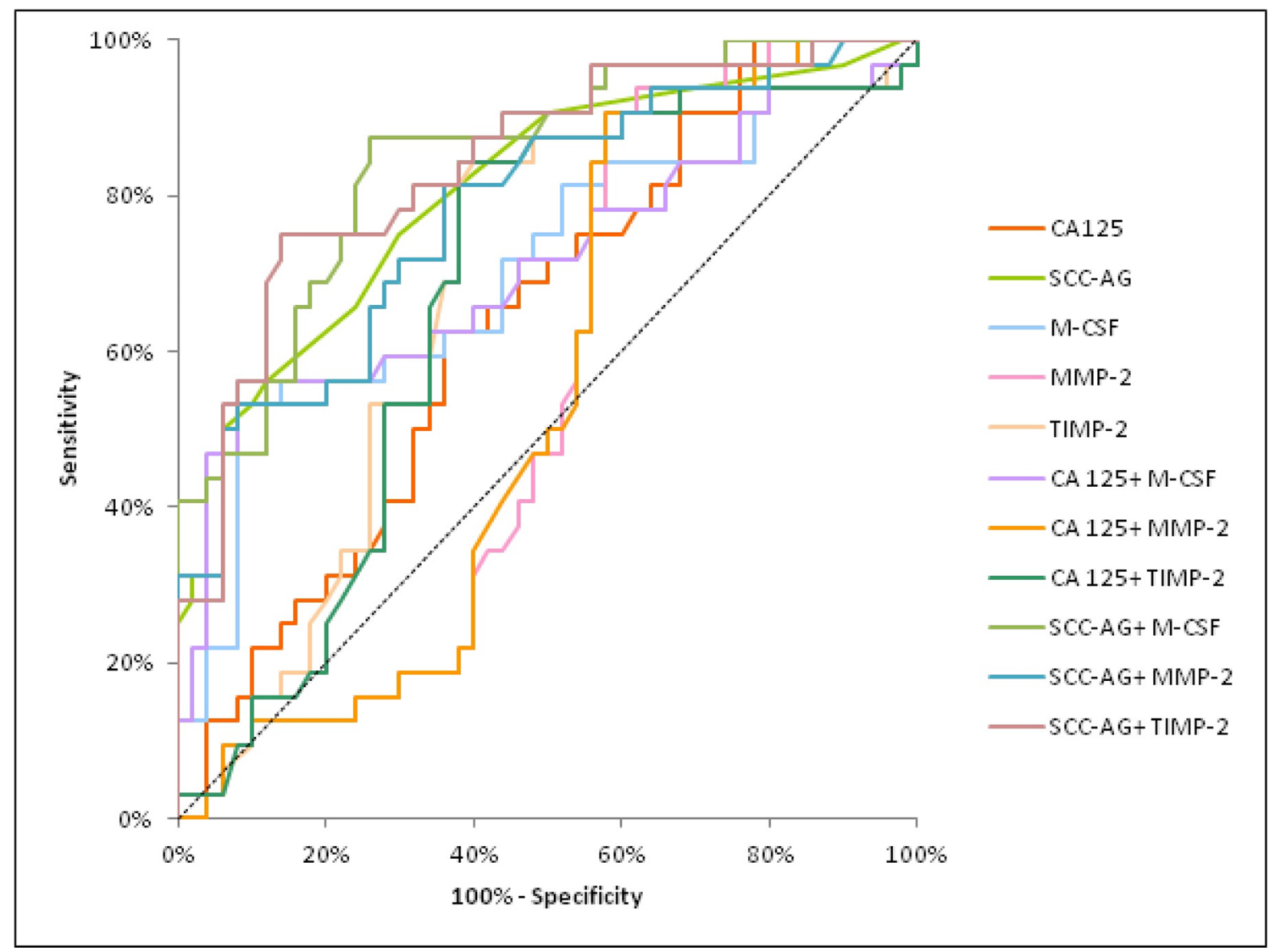

Fig. 2 Diagnostic criteria of ROC curve for examined parameters in combination with CA 125 and SCC-Ag in stage I of cervical cancer group. Abbreviations: ROC, receiver-operating characteristics; M-CSF, macrophage-colony stimulating factor; MMP-2, matrix metalloproteinase-2; TIMP-2, tissue inhibitor of metalloproteinase-2 
observed for the combination of MMP-2 with CA 125 in stages II, III and IV (100\% in all cases).

The diagnostic SP of the tested parameters was the highest for M-CSF (86\%) and was higher than that for CA $125(68 \%)$ and SCC-Ag (74\%).

The relationship between the diagnostic SE and SP is illustrated by the ROC curve (Tables 4 and 5). The AUC indicates the clinical usefulness of a tumor marker and its diagnostic power. We noticed that the AUC for M-CSF (0.8051) in the total CC group was larger than the area of SCC-Ag (0.7866), CA 125 (0.7340), TIMP-2 (0.6186) and MMP-2 (0.5882). Moreover, areas under the ROC curve for M-CSF and TIMP-2, similarly as for CA 125 and SCC-Ag, were statistically significantly larger in comparison to AUC $=0.5$ (borderline of the diagnostic usefulness of the test) $(p<0.001 ; p=$ $0.0257 ; p<0.001 ; p<0.001$; respectively) The combined analysis of AUC for M-CSF, MMP-2, and TIMP-2 with antigen $\mathrm{SCC}$ resulted in an increase in the areas in all cases (0.8760; 0.7880; 0.8081; respectively) (Fig. 1).

The AUC of M-CSF and MMP-2 demonstrated a distinct increase, concomitant with $\mathrm{CC}$ stage, identically to CA 125. In stage I of $\mathrm{CC}$ the highest AUC of all the tested parameters was found in SCC-Ag (0.8022) and it was the parameter which was statistically significantly larger in comparison to $\mathrm{AUC}=0.5(p<0.001)$, similarly to M-CSF $(p=0.0011)$, TIMP-2 $(p=0.0064)$ and CA 125 ( $p=0.0188)$ (Fig. 2). In stage II of CC the highest AUC of all the tested parameters was observed in M-CSF $(0.8042 ; p<0.001)$ and it was marginally higher than SCC-Ag (0.7973). Moreover, the AUCs for M-CSF and TIMP-2, similarly to those for CA 125 and SCC-Ag, were statistically significantly larger in comparison to $\mathrm{AUC}=0.5$ ( $p<0.001 ; p=0.0062 ; p<0.001 ; p<0.001$; respectively) (Fig. 3). In stages III and IV of CC the highest AUC of all the tested parameters was observed in M-CSF $(0.8797$; $p<0.001)$ and it was marginally higher than CA 125 (0.8114; $p<0.001)$. Additionally, the AUCs for M-CSF and MMP-2, similarly as for CA 125 and SCC-Ag, were statistically significantly larger in comparison to $\mathrm{AUC}=0.5$ ( $p<0.001 ; p=0.0098 ; p<0.001 ; p<0.001$; respectively). (Fig. 4). The combined analysis of AUC for the tested parameters (M-CSF, MMP-2 or TIMP-2) with antigen SCC resulted in an increase in the areas in all stages of $\mathrm{CC}(p<0.001)$.

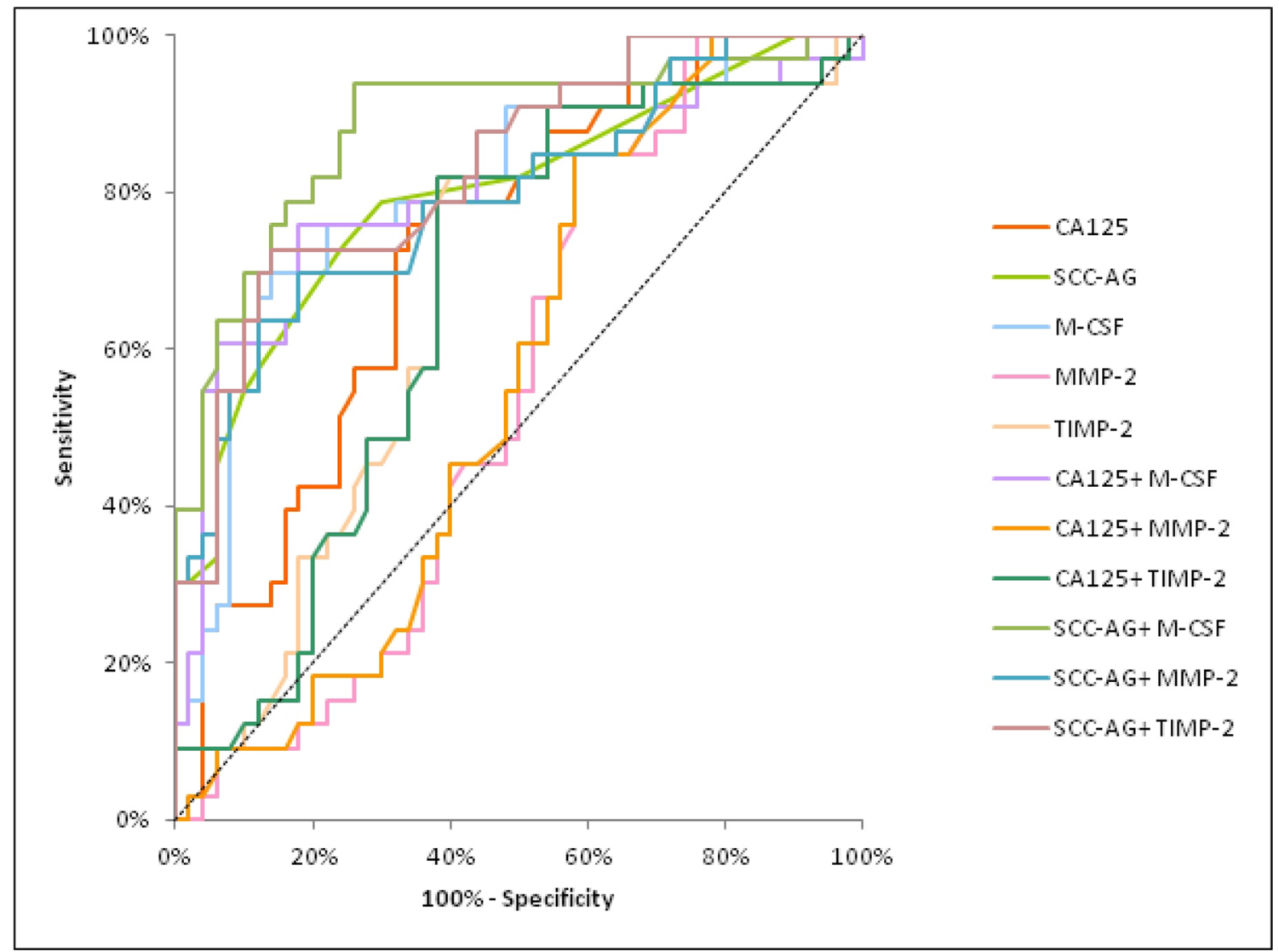

Fig. 3 Diagnostic criteria of ROC curve for examined parameters in combination with CA 125 and SCC-Ag in stage II of cervical cancer group. Abbreviations: ROC, receiver-operating characteristics; M-CSF, macrophage-colony stimulating factor; MMP-2, matrix metalloproteinase-2; TIMP-2, tissue inhibitor of metalloproteinase-2 


\section{Discussion}

The most promising markers appear to be serum cytokines, matrix metalloproteinases and their tissue inhibitors produced by a wide variety of cells, which play a central role in the regulation of inflammatory processes and the immune system. An ideal tumor marker should have a high sensitivity and specificity in order to discriminate between patients with cancer and those with benign conditions or healthy controls, and should also provide information related to tumor burden and activity [22]. Many new tumor markers have been discovered since the development of monoclonal antibodies, and the majority of tumors are now detected using them. Cytokines play an important role in tumor-stroma interaction, thus facilitating tumor progression and aggressiveness. Recombinant human M-CSF induces angiogenesis through macrophages by promoting VEGFA expression [23]. We have previously found increased concentrations of M-CSF in the plasma of patients with endometrial [24], breast [25] and ovarian cancer [26]. Moreover, M-CSF is a good candidate as a marker of colorectal [27] and gastric cancer [28]. A number of other authors have also pointed to the role of MMP-2 in cancer invasion and metastasis $[29,30]$. Matrix metalloproteinases are able to remodel the extracellular matrix (ECM). It has been indicated that this enzymes might be produced by tumor cells [31]. The activity of MMP-2 is mainly controlled by interactions with its natural inhibitor - tissue inhibitor of metalloproteinase-2 (TIMP2). The imbalance between these MMPs and TIMPs as a result of the increased production and activation of MMPs is responsible for cancer metastasis [31].

In the present study, the ELISA method was used to measure the plasma concentrations of M-CSF, MMP-2, TIMP-2 in cervical cancer patients. The levels of M-CSF, CA 125 and SCC-Ag were significantly higher compared to healthy subjects. Only the plasma levels of TIMP-2 were statistically significantly lower when compared to healthy controls. These findings are in agreement with our previous study showing that the median levels of M-CSF in cervical cancer patients are higher $(510.55 \mathrm{pg} / \mathrm{mL})$ than in healthy subjects (290.82 pg/mL) [32]. Our observation is supported by data regarding M-CSF from a previous report by Lawicki et al. $[33]$ in breast cancer patients. They are also in line with the observations of other authors studying M-CSF levels in patients with pancreatic cancer [34]. Furthermore, it has observed that M-CSF concentrations are statistically different in every group (the analysis related to CC stage) compared to healthy subjects. Similar results were obtained by Ławicki

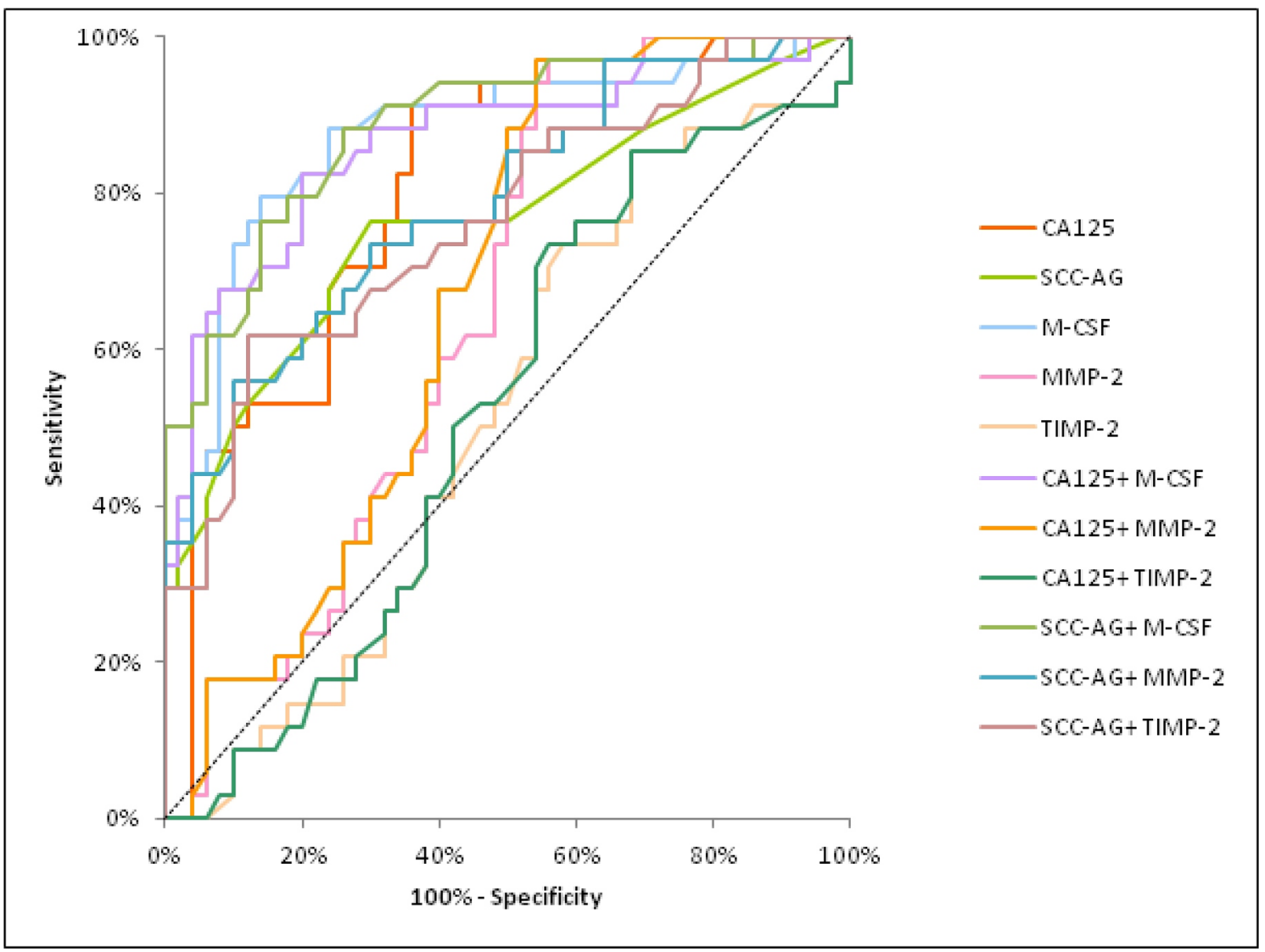

Fig. 4 Diagnostic criteria of ROC curve for examined parameters in combination with CA 125 and SCC-Ag in stages III and IV of cervical cancer group. Abbreviations: ROC, receiver-operating characteristics; $\mathrm{M}$ -
CSF, macrophage-colony stimulating factor; MMP-2, matrix metalloproteinase-2; TIMP-2, tissue inhibitor of metalloproteinase-2 
et al. [35] who observed higher concentrations of M-CSF in all the analysed groups in relation to breast cancer stage compared to healthy women. Significantly elevated levels of MCSF in advanced stages of cancer have also been found in patients with malignancies of the reproductive organs [8]. In our study, similar data were obtained for CA 125 (with the exception of stage I). Furthermore, elevated levels of M-CSF and CA 125 correspond to the positive correlations of M-CSF and CA 125 with more advanced stages of cervical cancer. Additionally, this study found high levels of MMP-2 in stages III and IV. The same observations regarding MMP-2 were reported by Ghosh et al. [36] who demonstrated that MMP-2 protein expression increased differentially in cervical cancer and it was associated with carcinoma stage.

In our study, the sensitivity of MMP-2 was the highest out of all the tested parameters (92.05\%). Ghosh et al. [36] also showed high sensitivity values of MMP-2 in cervical cancer patients, although that study employed zymographic analysis to measure MMP-2 in the samples of cervical tissue. In the present study, the combined use of M-CSF, MMP-2 or TIMP2 with the commonly accepted tumor markers resulted in an increase in SE values. Among all the parameters, the highest $\mathrm{SE}$ in all stages of cancer was observed for MMP-2 (93.10\%, $82.76 \%$; $96.88 \%$; respectively). In the publication by Ławicki et al. [33] concerning patients with breast cancer, the sensitivity of MMP-2 was lower than that reported in the present study. The highest values of SE were obtained for the combination of MMP-2 with the commonly accepted tumor markers in all stages of cancer. In our previous study, the highest values were observed for the combination of M-CSF, TIMP-2 and CA 15-3 in all stages of breast cancer group [33].

In this study, the SP of M-CSF $(86 \%)$ was the highest out of all the studied parameters and was higher than that of SCC-Ag (74\%) and CA 125 (68\%). In the case of M-CSF, the specificity reported in the paper by Ławicki et al. [37] on cervical cancer was marginally higher than that obtained in our study (92\%). MCSF specificity obtained by Vasiliades [34] et al. in the study on pancreatic cancer was lower than ours $(62.5 \%)$. This discrepancy is probably due to the differences in the types of cancers studied.

The area under the ROC curve indicates the clinical usefulness of a tumor marker. In this study, the area under the ROC curve of M-CSF was the largest out of all the tested parameters in the total CC group. Additionally, we observed statistically significantly larger AUCs for the tested parameters (with the exception of MMP-2), compared to AUC $=0.5$ (borderline of diagnostic usefulness of the test). The combined analysis of AUC for all the tested parameters with the commonly accepted tumor marker (SCC-Ag) resulted in an increase in the areas in all cases. A study by Ławicki et al. [32] reported the area under the ROC curve of M-CSF to be marginally lower than ours. Our results show that the diagnostic power (AUC) of the tested parameters, especially $\mathrm{M}-\mathrm{CSF}$, in the group of $\mathrm{CC}$ patients was marginally lower in comparison with the diagnostic power of M-CSF in the course of pancreatic cancer study conducted by Vasiliades et al. [34]. Moreover, the area under the ROC curve of M-CSF and the combined analysis of M-CSF with CA 125 and SCC-Ag were larger than the area of MMP-2 or TIMP-2 in all stages of cancer. In our previous study, we observed that M-CSF had the largest AUC in stage IV of breast cancer [35].

\section{Conclusions}

The findings of this study suggest the usefulness of M-CSF, MMP-2 and TIMP-2 in the diagnostics of CC patients, particularly in combination with CA 125 and SCC-Ag. The area under the ROC curve was highest for the combination of MCSF and the commonly accepted tumor markers, which indicates potential clinical significance of plasma M-CSF in the diagnosis of CC. Out of the tested substances, M-CSF also appears to be the best candidate for cancer diagnostics in all stages of the disease, based on ROC analysis.

\section{Compliance with Ethical Standards}

Conflict of Interest The authors of the article declare that they have no competing interests.

Abbreviations $C C$, Cervical cancer; $M-C S F$, Macrophage colonystimulating factor; $M M P$-2, Matrix metalloproteinase-2; TIMP-2, Tissue inhibitor of metalloproteinase-2

Open Access This article is distributed under the terms of the Creative Commons Attribution 4.0 International License (http:// creativecommons.org/licenses/by/4.0/), which permits unrestricted use, distribution, and reproduction in any medium, provided you give appropriate credit to the original author(s) and the source, provide a link to the Creative Commons license, and indicate if changes were made.

Publisher's Note Springer Nature remains neutral with regard to jurisdictional claims in published maps and institutional affiliations.

\section{References}

1. Torre LA, Bray F, Siegel RL, Ferlay J, Lortet-Tieulent J, Jemal A (2015) Global cancer statistics, 2012. CA Cancer J Clin 65(2):87108. https://doi.org/10.3322/caac.21262

2. Jin L, Xu ZX (2015) Recent advances in the study of HPVassociated carcinogenesis. Virol Sin 30(2):101-106. https://doi. org/10.1007/s12250-015-3586-3

3. Tsikouras P, Zervoudis S, Manav B, Tomara E, Iatrakis G, Romanidis C, Bothou A, Galazios G (2016) Cervical cancer: screening, diagnosis and staging. J BUON 21(2):320-325

4. Lewis CE, Pollard JW (2006) Distinct role of macrophages in different tumor microenvironments. Cancer Res 66(2):605-612. https://doi.org/10.1158/0008-5472.CAN-05-4005

5. Achkova D, Maher J (2016) Role of the colony-stimulating factor (CSF)/CSF-1 receptor axis in cancer. Biochem Soc Trans 44(2): 333-341. https://doi.org/10.1042/BST20150245

6. Zajkowska M, Głażewska EK, Będkowska GE, Chorąży P, Szmitkowski M, Ławicki S (2016) Diagnostic power of vascular 
endothelial growth factor and macrophage Colony-stimulating factor in breast Cancer patients based on ROC analysis. Mediat Inflamm 2016:5962946. https://doi.org/10.1155/2016/5962946

7. Richardsen E, Uglehus RD, Johnsen SH, Busund LT (2015) Macrophage-colony stimulating factor (CSF1) predicts breast cancer progression and mortality. Anticancer Res 35(2):865-874

8. Bedkowska GE, Ławicki S, Gacuta E, Pawłowski P, Szmitkowski M (2015) M-CSF in a new biomarker panel with HE4 and CA 125 in the diagnostics of epithelial ovarian cancer patients. J Ovarian Res 8:27. https://doi.org/10.1186/s13048-015-0153-3

9. Harano K, Hirakawa A, Kato T, Suzuki K, Watanabe S, Katsumata N (2014) Use of colony-stimulating factor in patients with ovarian cancer receiving paclitaxel and carboplatin in Japan. J Gynecol Oncol 25(2):124-129. https://doi.org/10.3802/jgo.2014.25.2.124

10. Poonawalla IB, Piller LB, Lairson DR, Chan W, Du XL (2016) Use of hematopoietic growth factors and risk of thromboembolic and pulmonary toxicities in elderly patients with advanced ovarian Cancer. Womens Health Issues 26(5):574-583. https://doi.org/10. 1016/j.whi.2016.05.007

11. Aharinejad S, Salama M, Paulus P, Zins K, Berger A, Singer CF (2013) Elevated CSF1 serum concentration predicts poor overall survival in women with early breast cancer. Endocr Relat Cancer 20(6):777-783. https://doi.org/10.1530/ERC-13-0198

12. Ławicki S, Głażewska EK, Sobolewska M, Będkowska GE, Szmitkowski M (2016) Plasma levels and diagnostic utility of macrophage Colony-stimulating factor, matrix Metalloproteinase-9, and tissue inhibitor of Metalloproteinases-1 as new biomarkers of breast Cancer. Ann Lab Med 36(3):223-229. https://doi.org/10. 3343/alm.2016.36.3.223

13. Rego SL, Helms RS, Dréau D (2014) Breast tumor cell TACE-shed MCSF promotes pro-angiogenic macrophages through NF- $\mathrm{KB}$ signaling. Angiogenesis 17(3):573-585. https://doi.org/10.1007/ s10456-013-9405-2

14. Björklund M, Koivunen E (2005) Gelatinase-mediated migration and invasion of cancer cells. Biochim Biophys Acta 1755(1):3769. https://doi.org/10.1016/j.bbcan.2005.03.001

15. Turpeenniemi-Hujanen T (2005) Gelatinases (MMP-2 and -9) and their natural inhibitors as prognostic indicators in solid cancers. Biochimie 87(3-4):287-297. https://doi.org/10.1016/j.biochi. 2005.01.014

16. Remillard TC, Bratslavsky G, Jensen-Taubman S, StetlerStevenson WG, Bourboulia D (2014) Molecular mechanisms of tissue inhibitor of metalloproteinase 2 in the tumor microenvironment. Mol Cell Ther 2:17. https://doi.org/10.1186/2052-8426-2-17

17. Walsh LA, Cepeda MA, Damjanovski S (2012) Analysis of the MMP-dependent and independent functions of tissue inhibitor of metalloproteinase- 2 on the invasiveness of breast cancer cells. J Cell Commun Signal 6(2):87-95. https://doi.org/10.1007/s12079011-0157-8

18. Chakraborti S, Mandal M, Das S, Mandal A, Chakraborti T (2003) Regulation of matrix metalloproteinases: an overview. Mol Cell Biochem 253(1-2):269-285

19. Bourboulia D, Stetler-Stevenson WG (2010) Matrix metalloproteinases (MMPs) and tissue inhibitors of metalloproteinases (TIMPs): positive and negative regulators in tumor cell adhesion. Semin Cancer Biol 20(3):161-168. https://doi.org/10.1016/j. semcancer.2010.05.002

20. Brew K, Nagase H (2010) The tissue inhibitors of metalloproteinases (TIMPs): an ancient family with structural and functional diversity. Biochim Biophys Acta 1803(1):55-71. https://doi.org/10. 1016/j.bbamcr.2010.01.003

21. Kähäri VM, Saarialho-Kere U (1999) Matrix metalloproteinases and their inhibitors in tumour growth and invasion. Ann Med 31(1):34-45

22. Zola P, Macchi C, Cibula D, Colombo N, Kimmig R, Maggino T, Reed N, Kesic V (2015) Follow-up in gynecological malignancies: a state of art. Int J Gynecol Cancer 25(7):1151-1164. https://doi. org/10.1097/IGC.0000000000000498

23. Eubank TD, Galloway M, Montague CM, Waldman WJ, Marsh CB (2003) M-CSF induces vascular endothelial growth factor production and angiogenic activity from human monocytes. J Immunol 171(5):2637-2643

24. Lawicki S, Bedkowska E, Gacuta-Szumarska E, Czygier M, Szmitkowski M (2007) The plasma levels and diagnostic utility of stem cell factor and macrophage-colony stimulating factor in endometrial cancer patients. Przegl Lek 64(12):987-990

25. Ławicki S, Szmitkowski M, Wojtukiewicz M (2006) The pretreatment plasma level and diagnostic utility of M-CSF in benign breast tumor and breast cancer patients. Clin Chim Acta 371(1-2):112116. https://doi.org/10.1016/j.cca.2006.02.033

26. Lawicki S, Gacuta-Szumarska E, Bedkowska GE, Szmitkowski M (2012) Hematopoietic cytokines as tumor markers in gynecological malignancies. A multivariate analysis in epithelial ovarian cancer patients. Growth Factors 30(6):357-366. https://doi.org/10.3109/ 08977194.2012.724407

27. Mroczko B, Szmitkowski M, Wereszczyńska-Siemiatkowska U, Okulczyk B, Kedra B (2007) Pretreatment serum levels of hematopoietic cytokines in patients with colorectal adenomas and cancer. Int $\mathrm{J}$ Color Dis 22(1):33-38. https://doi.org/10.1007/s00384-006-0099-4

28. Liu H, Zhang H, Shen Z, Lin C, Wang X, Qin J, Qin X, Xu J, Sun Y (2016) Increased expression of CSF-1 associates with poor prognosis of patients with gastric Cancer undergoing gastrectomy. Medicine (Baltimore) 95(9):e2675. https://doi.org/10.1097/MD. 0000000000002675

29. Fingleton B (2006) Matrix metalloproteinases: roles in cancer and metastasis. Front Biosci 11:479-491

30. Pellikainen JM, Ropponen KM, Kataja VV, Kellokoski JK, Eskelinen MJ, Kosma VM (2004) Expression of matrix metalloproteinase (MMP)-2 and MMP-9 in breast cancer with a special reference to activator protein-2, HER2, and prognosis. Clin Cancer Res 10(22): 7621-7628. https://doi.org/10.1158/1078-0432.CCR-04-1061

31. Crawford HC, Matrisian LM (1994) Tumor and stromal expression of matrix metalloproteinases and their role in tumor progression. Invasion Metastasis 14(1-6):234-245

32. Ławicki S, Będkowska GE, Gacuta-Szumarska E, Knapp P, Szmitkowski M (2012) Pretreatment plasma levels and diagnostic utility of hematopoietic cytokines in cervical cancer or cervical intraepithelial neoplasia patients. Folia Histochem Cytobiol 50(2):213-219

33. Ławicki S, Zajkowska M, Głażewska EK, Będkowska GE, Szmitkowski M (2016) Plasma levels and diagnostic utility of MCSF, MMP-2 and its inhibitor TIMP-2 in the diagnostics of breast Cancer patients. Clin Lab 62(9):1661-1669

34. Vasiliades G, Kopanakis N, Vasiloglou M, Zografos G, Margaris H, Masselou K, Kokosi E, Liakakos T (2012) Role of the hematopoietic cytokines SCF, IL-3, GM-CSF and M-CSF in the diagnosis of pancreatic and ampullary cancer. Int J Biol Markers 27(3):e186e194. https://doi.org/10.5301/JBM.2012.9348

35. Ławicki S, Bẹdkowska GE, Szmitkowski M (2013) VEGF, M-CSF and CA 15-3 as a new tumor marker panel in breast malignancies: a multivariate analysis with ROC curve. Growth Factors 31(3):98105. https://doi.org/10.3109/08977194.2013.797900

36. Ghosh A, Moirangthem A, Dalui R, Ghosh T, Bandyopadhyay A, Dasgupta A, Banerjee U, Jana N, Basu A (2014) Expression of matrix metalloproteinase-2 and 9 in cervical intraepithelial neoplasia and cervical carcinoma among different age groups of premenopausal and postmenopausal women. J Cancer Res Clin Oncol 140(9):1585-1593. https://doi.org/10.1007/s00432-014-1695-2

37. Lawicki S, Bedkowska E, Gacuta-Szumarska E, Knapp P, Szmitkowski M (2008) The plasma levels and diagnostic utility of stem cell factor (SCF) and macrophage-colony stimulating factor (M-CSF) in cervical cancer patients. Pol Merkur Lekarski 25(145): $38-42$ 